\title{
Neuroendocrine tumor of appendix presenting as appendicitis
}

\begin{abstract}
Neuroendocrine tumors (NET) of the appendix are rare and is usually detected incidentally after an appendectomy. ${ }^{1}$ They are derived from the subepithelial cell population. If the tumor is less than $1 \mathrm{~cm}$ then it can be treated with appendectomy with no further treatment required. However if the tumor is greater than $2 \mathrm{~cm}$ then right hemicolectomy needs to be performed due to the significant risk for the metastasis. Overall prognosis of the NET of the appendix in all ages is excellent. ${ }^{1}$ We present a case of a 34yr old male who presented to the emergency room with persistent RLQ pain for several weeks with clinical findings suggesting appendicitis.
\end{abstract}

Keywords: appendicitis, non-epithelial tumors, neuroendocrine tumors, persistent RLQ pain
Volume II Issue I - 2020

\author{
Chintav Shah,' Jay Patolia,' Simeon \\ Jaggernauth, ${ }^{2}$ Nirav Nagarsheth' \\ 'Department of Internal Medicine, University of Arkansas for \\ Medical Sciences, USA \\ ${ }^{2}$ Landmark Cancer Center,Arkansas, USA
}

Correspondence: Chintav Shah, Department of Interna Medicine, University of Arkansas for Medical Sciences, Fayetteville, Arkansas, USA,

Email Chintavsha65@gmail.com,chsha@uams.edu

\section{Background}

The term "carcinoid" was first described in 1907 to describe the small benign little carcinoma of the small intestine. ${ }^{1}$ The term carcinoid was later replaced by neuroendocrine tumors. The most common presentation is incidental finding on appendectomy. It usually appears at the tip of the appendix. ${ }^{1}$ They are rare with 1.2 cases seen per 100,000 in the United States. ${ }^{2}$

Tumors of the appendix can be divided into epithelial and nonepithelial tumors. Epithelial tumors are adenocarcinomas while most of the non-epithelial tumors are neuroendocrine tumors. ${ }^{3,4}$

\section{Case report}

This was 34yr old male with no PMH was in his usual state of health at work loading a truck where he experienced a fall resulting in persistent RLQ pain. He had a CT scan of the abdomen and pelvis performed that was normal with a non visualised appendix. Patient was seen by surgery who suggested an appendectomy however the patient wasn't ready for it and wanted to try antibiotics. He was placed on ciprofloxacin and metronidazole. His symptoms worsened therefore he returned to the emergency room and was admitted. He underwent a laparoscopic appendectomy with no visible tumor seen at the time of the surgery.

Pathology returned with the evidence of a well differentiated NET of the appendix measuring $2 \mathrm{~mm}$ with a mitotic rate of 0 located at the tip of the appendix. The tumor extended into muscularis wall with no extension into the serosa. Margins were negative and the lymph nodes were identified. He was classified as pathological T1. Immunohistochemical stain showed tumor cells positive for chromogranin and pancytokeratin. ${ }^{5}$

Patient was evaluated by oncology for the possible right hemicolectomy due to increased chance of metastasis. However with the tumor size being $2 \mathrm{~mm}$, clinical observation for $5 \mathrm{yrs}$ was recommended with baseline chromogranin $\mathrm{A}$ and serotonin to be followed along with regular CT scan of the abdomen and pelvis. His complaints or right lower quadrant pain significantly improved after removal of the appendix.

\section{Discussion}

The presentation of this patient was really different since the tumor size was so small and that small of a tumor would rarely cause such a severe presentation. The differentials for RLQ pain are vast and a neuroendocrine tumor would be the lowest on differentials. However with this case, with a normal CT and ultrasound of the abdomen, neuroendocrine tumor causing RLQ pain should be considered higher on the differential list. Appendectomy is the treatment of choice for tumors $<1 \mathrm{~cm}$. For tumors $>2 \mathrm{~cm}$, the North American Neuroendocrine Society (NANETS) suggest a right hemicolectomy for tumors originating from the base of the appendix, or there is evidence of metastasis or lymphovascular invasion. ${ }^{6}$

\section{Acknowledgments}

None.

\section{Conflicts of interest}

There is no conflict of interests regarding the publication of this case report.

\section{References}

1. Griniatsos J, Michail O. Appendiceal neuroendocrine tumors: recent insights and clinical implications. World $J$ Gastrointest Oncol. 2010;2(4):192-196.

2. Clinics in Colon and Rectal Surgery. Thieme E-Journals. 2015.

3. Alexandraki KI, Kaltsas GA, Grozinsky-Glasberg S, et al. Appendiceal neuroendocrine neoplasms: diagnosis and management. Endocr Relat Cancer. 2016;23(1):R27-R41. 
4. Shankar S, Ledakis P, El Halabi H, et al. Neoplasms of the appendix: current treatment guidelines. Hematol Oncol Clin North Am. 2012;26(6):1261-1290.

5. Kelly KJ. Management of appendix cancer. Clin Colon Rectal Surg. 2015;28(4):247-255.
6. Elkbuli A, Sanchez C, McKenney M, et al. Incidental neuro-endocrine tumor of the appendix: case report and literature review. Ann Med Surg (Lond). 2019;43:44-47. 